\title{
IR thermography assisted control for apples microwave drying
}

\author{
by G. Cuccurullo, L. Giordano and D. Albanese
}

*Dept. of Industrial Engineering, University of Salerno, Via Ponte Don Melillo- 84084 Fisciano (SA), Italy, gcuccurullo@unisa.it

\begin{abstract}
Microwave drying of sliced apples was carried out while fixing their temperature level in a multimode cavity. The temperature of the samples was controlled on-line through an infrared thermography assisted control system, that enabling to suitably adjust the delivered microwave power. The control strategy was no more related to a single arbitrary chosen slice but to the actual hottest one. The effectiveness of the system in realizing temperature was proven and drying kinetics were analyzed through the determination of the effective mass diffusivity by applying a 2D unsteady analytical model. Data reduction by the Levenberg Marquadt technique led to quite satisfactory agreement with analytical predictions.
\end{abstract}

\section{Introduction}

Drying is a widely used technique for food preservation, but long drying times and high temperatures are required by using sunlight or conventional hot air drying. Conventional drying turns out to be a low efficient process because of the foods low thermal conductivity and high internal resistance to moisture transfer [1-2]. Thus an increasing demand for faster procedures is arising in the food industry. In this connection, microwave (MW) driven drying processes have been studied extensively in the last decade since it is well known that they provide an efficient mean for moisture removal. In facts, it has been demonstrated that, for a large variety of foods, microwave heating considerably lowers drying times without damaging flavour, colour, nutrients of the fruit [3-12]. Improved drying rates can be explained considering that the interior of the sample is quickly heated by internal heat generation due to MW exposition and, as a consequence, the generated vapour is forced towards the surface where it is easily removed by the air flow. Furthermore, fast vapour expulsion creates a porous structure which facilitates the transport of vapour being responsible of reduced shrinkage [13-16]. On the other hand, some critical points arise when performing microwave heating, such as uneven temperature patterns as well as temperature readout and control problems. The former are due to electromagnetic fields behaviour which is highly uneven and hardly predictable while the latter are to be related to difficulties in measuring temperatures inside the illuminated cavity where traditional probes fails. As a matter of facts, typically, drying processes are carried-on fixing power rather than temperature levels: this occurrence can lead to product overheating, with charring and unacceptable final quality [13;17-22]. Thus, it is clear that temperature must be controlled [21-25] but such procedure is seldom reported in the literature [26-27], probably for the reasons outlined before.

To address the problem, a new microwave drying system was developed which can automatically and continuously adjust the power levels by acting on the magnetron duty cycle time in order to control the product temperature. The signal for realizing feedback temperature control was obtained looking inside the illuminated MW chamber by an IR temperature equipment; the latter allowed to detect the instantaneous maximum temperature among the sample slices distributed randomly on the turning table. For the first time the feedback signal was no more related to a single arbitrarily chosen slice inside the MW chamber, which can affect results [28], but to the actual hottest one. Air temperature, humidity and speed were also controlled while the sample-holding turntable was suspended to a technical balance located on the top of the oven for online mass measurement. Considering that microwave drying kinetics mainly depend on moisture diffusion phenomena since external and internal heating resistances become irrelevant to heat transfer mechanism, the ability of realizing experimental drying texts featured by constant temperature levels suggested to process the weight loss data by using a 2D unsteady analytical model for mass diffusion inside isothermal samples. Thus, the effective mass diffusivity [28-30] was determined and, as a consequence, the drying process prediction was allowed.

\section{Experimental setup}

Drying was performed in a pilot plant, Figure 1, projected for general purposes in order to encompass different loads, i.e. different materials and samples distributions, weight, size. Hence, it has been aimed that the illuminated cavity could behave as a reverberating chamber, at least in the central space, due to the superposition of multiple modes. A large (with respect to volume where the samples under test were placed) cavity was then required, thus generated microwaves illuminate an insulated metallic cubic chamber $\left(1 \mathrm{~m}^{3}\right)$. The microwave pilot plant was equipped with a 


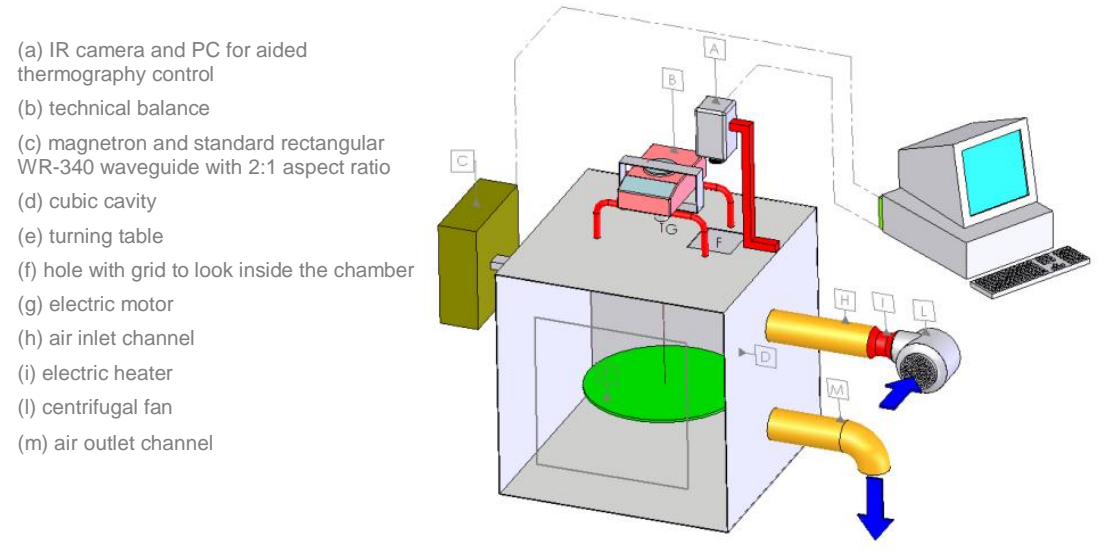

Fig. 1. Microwave pilot plant heating system

magnetron rated at $2 \mathrm{~kW}$, emitting at a frequency of $2.45 \mathrm{GHz}$. A mode stirrer rotating at 250 rpm was employed to enhance the microwave power distribution inside the cavity.

A teflon rotating tray ( $50 \mathrm{~cm}$ diameter, $22 \mathrm{rpm}$ ) housed the samples under test and was connected to a technical balance (Gibertini EU-C 1200 RS) located on the top of the oven. The balance allowed real-time mass measurements. The tray was rotated at $10 \mathrm{rpm}$ on a ball bearing shaft driven by an electrical motor.

An external centrifugal fan facilitated the moisture removal by forcing external air into the cavity; the renewal air flow was kept constant throughout the experiments by controlling the inverter feeding the fan electric motor. The channel feeding the external air flow was equipped with an electric heater controlled by a thermocouple placed on the air expulsion channel. The relative humidities and temperatures both of the exhaust and external air were measured by hygrocapacitative sensors (BABUC/M, LSI LASTEM Srl). A suitable combination of electric heater power and renewal airflow was selected in order to realize a fixed temperature level inside the illuminated chamber, with a reduced gap (about $10^{\circ} \mathrm{C}$ ) between inlet and ambient air temperatures.

An IR equipment (thermaCAM flir P65) looked inside the oven through a hole $70 \mathrm{~mm}$ diameter properly shielded with a metallic grid trespassed by infrared radiation arising from the detected scene but impermeable to high-length EM radiation produced by the magnetron. The camera was calibrated to account the presence of grid as follows: the temperatures of a large apple slice, cooled from $90{ }^{\circ} \mathrm{C}$ to $35^{\circ} \mathrm{C}$, were simultaneously recorded by the IR equipment and by a thermocouple placed on the surface of the target. The emissivity of the target was set to 1, while the air temperatures inside and outside the cavity were set to the same levels adopted during the experiments; thus the reflected ambient temperature was fixed and IR thermography readout was allowed. A second order polynomial related the two measured temperatures.

The measured data, i.e. samples weight, internal and external air temperature, the reflected magnetron energy and duty cycle, were transferred to a personal computer each minute for control and recording purposes. Power control based on thermography temperature readout was accomplished governing an I/O board (AT MIO 16XE50, National Instruments, TX, USA) with a suitably developed Lab View 7.1 code (National Instruments, TX, USA). In particular, the instantaneous maximum temperature in the detected scene was communicated via RS232 to the controlling code each 0.7 seconds.

\section{Experimental procedure}

Preliminary experimental tests were carried on white apples (Golden delicious); their initial moisture content was $86 \%$ wet base. Apples were handy peeled, shaped as cylindrical pieces with a diameter of $20 \mathrm{~mm}, 10 \mathrm{~mm}$ height, and finally distributed on the tray. The total amount of samples considered for each trial was $300 \mathrm{~g}$ that resulting in $6.7 \mathrm{~W} / \mathrm{g}$ energy density. Weight changes during MW drying were registered on line by the technical balance.

The IR system acquired the instantaneous surface temperature map inside the illuminated cavity gathering in the IR scene a number of samples covering about $20 \%$ (see Figure 2). The code scanned for the maximum temperature among the samples appearing in the IR image under test. Because of the turntable rotation, different samples were individuated by analyzing different images. Then code automatically adjusted the magnetron delivered power by operating it in intermittent mode with constant power density; an on-off control was then set inside a specified differential band $\left( \pm 1^{\circ} \mathrm{C}\right)$ over the target temperature. The code also deliberated the end of the experimental test when the actual moisture content achieved a conventional fixed value of $20 \%$.

Three selected temperature levels were chosen for processing the samples under test, namely 55,65 and $75{ }^{\circ} \mathrm{C}$; for all of them the internal air temperature was set to $30^{\circ} \mathrm{C}$ with an air speed equal to $2.5 \mathrm{~m} / \mathrm{s}$ on the tray layer. The external relative humidity was controlled by an air conditioning system so to realize $6 \mathrm{~g}_{\mathrm{w}} / \mathrm{kg}_{\mathrm{a}}$. The renewal air flow was 

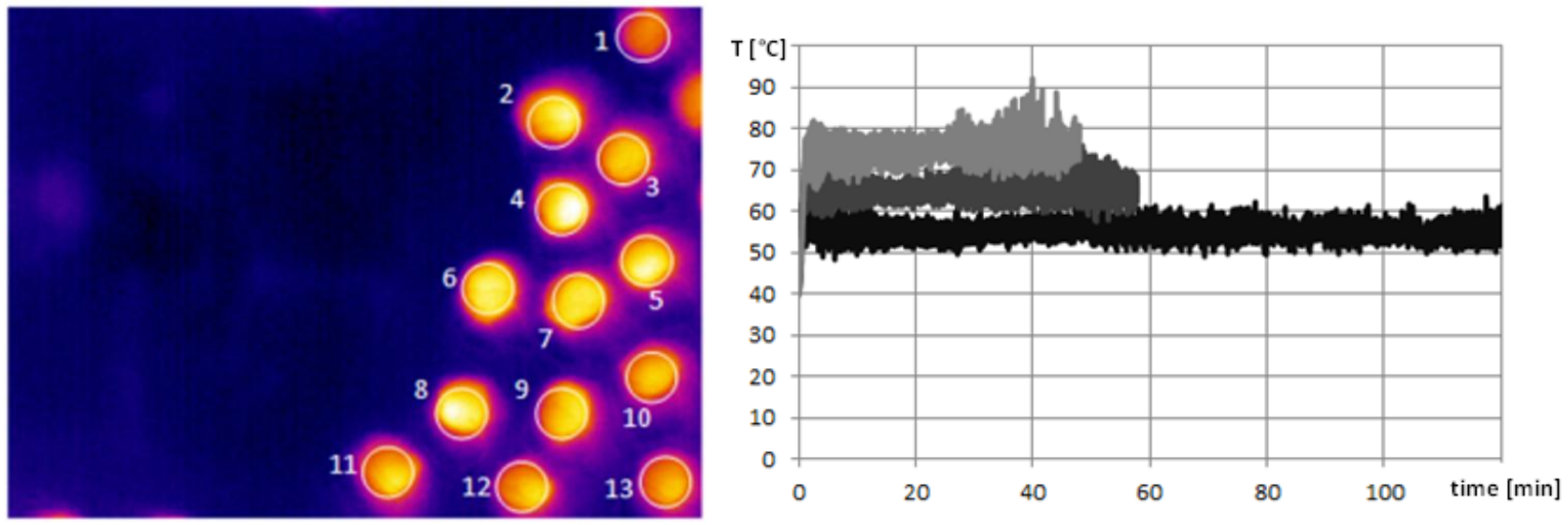

Fig. 2. Real-time IR thermography for apple slices

Fig. 3. Temperature fluctuations for the selected temperature levels

fixed to $25 \mathrm{~m}^{3} / \mathrm{h}$ in order to contain the inlet temperature excess within few degrees. All the experiments were repeated in triplicates and the related average values were processed.

The ability of the system at hand to keep the controlled temperature at the desired values, is shown in Figure 3. It can be easily checked that the overheating which features the uncontrolled MW heating-up period was avoided; however, temperature fluctuations become larger with decreasing moisture content. As expected, temperature span also increases with increasing temperature levels due to the decreasing of the dielectric loss factor with temperature.

\section{Basic Equations}

Several models have been proposed to describe the rate of water loss during drying processes [6; 31-37]. Among them, the description in terms of the effective diffusivity appears to be more adequate than the ones steaming from empirical kinetics: in facts, the latter don't exhibit a general validity being related to specific load and boundary conditions features [38]. At the aim of determining the effective diffusivity, the decrease of water in the apple slices has been described trough an analytical model based on the local mass balance assuming isothermal conditions, constant properties, negligible shrinking and cylindrical shapes for samples, Figure 2. Considering first type boundary conditions, the dimensionless equations turn out to be:

$$
\frac{\partial \mu}{\partial r}=\left[\frac{1}{r} \cdot \frac{\partial}{\partial r}\left(r \cdot \frac{\partial \mu}{\partial r}\right)+\frac{R_{0}^{2}}{L^{2}} \frac{\partial^{2} \mu}{\partial z^{2}}\right]
$$

$\mu(z=1, r, T)=0$

$\mu(z, r=1, T)=0$

$\left.\frac{\partial \mu}{\partial z}\right|_{z=0, r}=0$

$\left.\frac{\partial \mu}{\partial z}\right|_{r, z=0}=0$

$\mu(z, r, \tau=0)=1$

where: $\mu(z, r, \tau)=\left(x(z, r, \tau)-x_{\mathrm{e}}\right) /\left(x_{0}-x_{\mathrm{e}}\right)$ is the normalized moisture ratio, $x$ being the mass fraction of the water on dry basis; $x_{\mathrm{e}}$ and $x_{0}$ are the unperturbed air and the initial mass fractions of the water in the apple slices; $z=Z / L$ and $r=R / R_{0}$ are the dimensionless axial and radial coordinates, $2 L$ and $R_{0}$ being the height and the radius of the cylinder, respectively; $\tau=t / t_{0}$ is the dimensionless time, with $t_{0}=R_{0}{ }^{2} / D$.

The problem being linear and homogeneous, the solution of the problem may be written as the product of two partial solutions, each one depending on a single spatial coordinate, $\mu(z, r, \tau)=\mu_{1}(z, \tau) \cdot \mu_{2}(r, \tau)$. The two partial solutions have to 


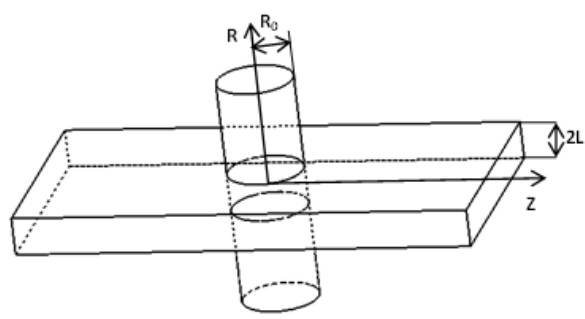

Fig. 4. Sample geometry

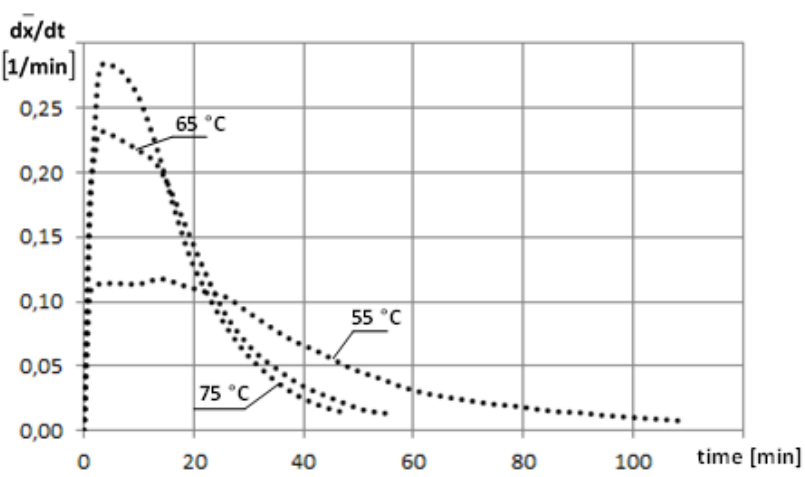

Fig. 5 Drying rate curves

satisfy two distinct problems:

$$
\begin{aligned}
& \frac{\partial \mu_{1}}{\partial \tau}=\frac{\mathrm{R}_{0}^{2}}{\mathrm{~L}^{2}} \frac{\partial^{2} \mu_{1}}{\partial z^{2}} \\
& \mu_{1}(z=1, \tau)=0 \\
& \left.\frac{\partial \mu_{1}}{\partial z}\right|_{z=0, \tau}=0 \\
& \mu_{1}(z, \tau=0)=1
\end{aligned}
$$

$$
\begin{aligned}
& \frac{\partial \mu_{2}}{\partial \tau}=\frac{1}{r} \cdot \frac{\partial}{\partial r}\left(r \cdot \frac{\partial \mu_{2}}{\partial r}\right) \\
& \mu_{2}(r=1, \tau)=0 \\
& \left.\frac{\partial \mu_{2}}{\partial r}\right|_{r=0, \tau}=0 \\
& \mu_{2}(r, \tau=0)=1
\end{aligned}
$$

Both the two sub-problems are well-known, the former being related to the infinite slab, the latter to the infinite cylinder; they were solved by the separation of variables method yielding:

$$
\begin{aligned}
& \mu_{1}(z, \tau)=\sum_{\mathrm{n}=1}^{\infty} c_{\mathrm{n}} \cdot \mathrm{G}_{\mathrm{n}}(z) \cdot \operatorname{Exp}\left(-\lambda_{\mathrm{n}}^{2} \cdot \tau\right) \\
& \mu_{2}(r, \tau)=\sum_{\mathrm{n}=1}^{\infty} d_{\mathrm{n}} \cdot \mathrm{F}_{\mathrm{n}}(r) \cdot \operatorname{Exp}\left(-\beta_{\mathrm{n}}^{2} \cdot \tau\right)
\end{aligned}
$$

where $G_{n}(z)=\operatorname{Cos}\left[\lambda_{n}\left(L / R_{0}\right) z\right]$ and $F_{n}(r)=\operatorname{BesselJ_{0}}\left(\beta_{n} r\right)$ are the eigen functions and $\lambda_{n}, \beta_{n}$ are the related eigenvalues arising from the characteristics equations: $\operatorname{Cos}\left[\lambda_{\mathrm{n}}\left(L / R_{0}\right)\right]=0, \operatorname{Bessel} \mathrm{J}_{0}\left(\beta_{\mathrm{n}}\right)=0$.

\section{Data reduction}

Assuming that the measured weight loss was equally distributed for all the samples, it was possible to evaluate the drying curves for the three selected temperature levels. Evaluating their slopes the drying rate curves were built as reported in Figure 5. As expected, drying was faster at higher temperatures, but it became less sensitive with increasing temperature levels: slight differences can be appreciated between 65 and $75^{\circ} \mathrm{C}$ related curves, while the process at 55 ${ }^{\circ} \mathrm{C}$ is much slower. All the curves exhibit the three typical phases for fruit drying: the first one, roughly corresponding to the thermal transient and thus termed warming up period, where increasing moisture removal rates were realized; the second one, identified in correspondence of a constant rate of moisture removal; the third one, in which moisture rates fell off continuously [13;39].

Data reduction was carried-on by processing the falling rate period, when the resistance to species transfer by diffusion in the product is much larger than the resistance to species transfer by convection at the surface. Such an occurrence can be shown evaluating the mass transfer Biot number, $\mathrm{Bi}=\left(\mathrm{h}_{\mathrm{m}} L_{0}\right) / D$, where $\mathrm{h}_{\mathrm{m}}$ is the mass transfer coefficient, $L_{0}$ is the reference spatial coordinate. In the case of the air, the mass transfer coefficient can be estimated as $h_{m}=h /(\rho c), 1 / h$ being the thermal resistance to heat transfer, $\rho$ and $c$ being the mass density and the specific heat of the air [40]. The magnitudo order of the reference length and of the mass diffusivity is $10^{-2}$ and $10^{-9}$, respectively, thus the Biot number turns out to be much larger than unity. Accordingly, first type conditions on the wall are properly involved in equations (2) and (3) [41]. On the other hand, the use of the above model including the spatial dependence rather than a concentrated parameters model, was suggested by considering that the time extend needed for the apple moisture content to decrease from 86 to $20 \%$ is small when compared to the sample characteristics time, $R_{0}^{2} / D$. 


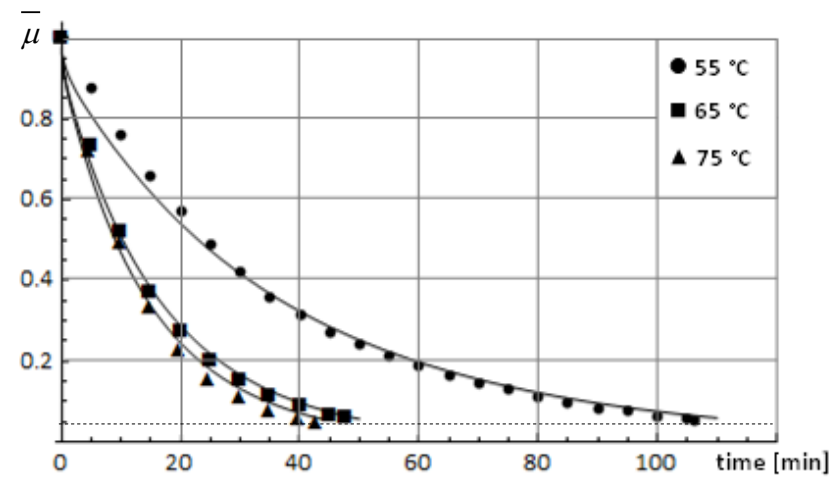

Fig. 6. Analytical prediction (continuous lines) vs experimental trend (falling rate period)

When performing measurements, it was assumed that the average weight loss for samples under test was described by the above model. Due to the complexity of the response model, the Levenberg Marquadt technique based fitting method has been selected. The technique enables to process non-linear models with an arbitrary number of parameters. Thus, the optimal choice for matching experimental and theoretical data was accomplished by minimizing the RMSE merit function:

$$
R M S E=\left[\frac{1}{N} \cdot \sum_{i=1}^{N}\left(\bar{\mu}\left(t_{i}, D\right)-\bar{\mu}_{i}\right)^{2}\right]^{1 / 2}
$$

where $\left(\mu_{i}, t_{i}\right)$ are the $N$ experimental normalized moisture content taken at the corresponding times $t_{i}$, the function $\bar{\mu}$ is the functional relationship given by the model for the average normalized moisture ratio, $D$ being the unknown diffusivity. The number of terms in the sum needed to evaluate $\bar{\mu}$ was chosen when the corresponding RMSE variation, $\Delta$, was less than 0.001.

In particular, moisture evolution of the samples was evaluated from the knowledge of the samples weight loss, $\Delta W$, and initial weight, $W_{0}$, such as follows:

$$
\mu=1-\frac{\Delta W}{W_{0}}\left(\frac{1+x_{0}}{x_{e}+x_{0}}\right) \cong 1-\frac{\Delta W}{W_{0}}\left(\frac{7.14}{6.14}\right)
$$

The above simplification is suggested by considering that the air humidity concentration is much smaller than the one in the food.

\section{Results and discussions}

Experimental curves and the corresponding analytical ones resulting from data reduction are reported in Fig. 6 for the three temperature levels at hand. The maximum standard deviation of the experimental data was equal to 0.28 $\mathrm{g}_{\text {water }} / \mathrm{g}_{\text {dry matter. }}$ A satisfying agreement between experimental and analytical data exists and is confirmed by the corresponding correlation coefficients, as shown in Table 1. In the same Table, the resulting diffusivities are reported; as expected, the effective mass diffusivity increases with temperature increasing while quantitative results seem to be consistent with the ones reported in literature. In particular, the values of apple effective diffusivities reported by some authors ranges from $1.1 \cdot 10^{-9}$ to $6.4 \cdot 10^{-9}$ depending on airflow and air temperature conditions [29;42-44]; however, the results presented in this work should be more appropriate since the analytical model holds strictly for isothermal conditions. Such occurrence is closely realized by the present experimental set-up rather than by traditional hot-air heating or by microwave heating without temperature control. In the Table 1 are also reported the number of terms needed in the sum and the corresponding RMSE variation.

\section{Conclusions}

Drying kinetics in a microwave controlled-temperature system have been studied for cylindrical apple samples. IR temperature detection allowed to keep constant values for sample surface temperature avoiding to establish control temperature in an arbitrarily fixed apple slice. If one considers that the electromagnetic field can be highly uneven in the illuminated cavity, operating temperature control in such a way can remove singular effects typically due to the fiber optic 
probe positioning thus assuring higher uniformity in the quality of the final product. The consequence of increasing temperature levels was to reduce drying times but with decreasing sensitivities. An isothermal 2D unsteady model for mass transfer was employed to describe the measured drying kinetics of apples. Quite satisfying results were obtained applying the model to recover experimental data probably due to operating at fixed temperature levels for the samples under test. Further test are intended to study the effect of different air velocities and temperatures, thus configuring combined microwave and convective heating for enhanced drying times.

Table 1. Data reduction results

\begin{tabular}{|c|c|c|c|c|c|}
\hline $\mathrm{T}\left[{ }^{\circ} \mathrm{C}\right]$ & $\mathrm{D}\left[\mathrm{m} / \mathrm{s}^{2}\right]$ & Terms & RMSE & $\Delta 10^{4}$ & Correlation Coeff. \\
\hline $55^{\circ} \mathrm{C}$ & $2.502 \cdot 10^{-9}$ & 10 & 0.10932 & 6.426 & 0.992 \\
\hline $65^{\circ} \mathrm{C}$ & $5.601 \cdot 10^{-9}$ & 16 & 0.07945 & 8.821 & 0.995 \\
\hline $75^{\circ} \mathrm{C}$ & $6.401 \cdot 10^{-9}$ & 12 & 0.14421 & 8.306 & 0.991 \\
\hline
\end{tabular}

\section{REFERENCES}

[1] Duygu Evin, "Microwave drying and moisture diffusivity of white mulberry: experimental and mathematical modeling", Journal of Mechanical Science and Technology, 25 (10) (2011)

[2] M. Maskan ans F. Gogus, "Sorption isotherms and drying characteristics of mulberry (Morus alba)", J. Food Eng., 37 (1998).

[3] N. R. Pereira, A. Marsaioli Jr., L. M. Ahrne, Effect of microwave power, air velocity and temperature on the final drying of osmotically dehydrated bananas, Journal of Food Engineering, 81 (2007)

[4] Alibas," Microwave, air and combined microwave - air drying parameters of pumpkin slices", LWT, 40 (2007)

[5] G. Dadali, D. Kılıç Apar and B. Özbek, "Microwave drying kinetics of okra", Dry. Tech. 25 (2007)

[6] M. Al-Harahsheh, A. H. Al-Muhtaseb, and T. R. A. Magee, "Microwave drying kinetics of tomato pomace: Effect of osmotic dehydratation", Chem. Eng. Process, 48 (2009).

[7] J. Wang and K. Sheng, "Far Infrared and microwave drying of peach", LWT Food Science and Technology, 39 (2006).

[8] Z. Wang, J. Sun, F. Chen, X. Liao and X. Hu, "Mathematical modeling on thin layer microwave drying of apple pomace with and without hot air pre-drying", Journal of Food Engineering, 80 (2007).

[9] B. Ozbek and G. Dadali, "Thin layer drying characteristics and modeling of mint leaves undergoing microwave treat\& ment, Journal of Food Engineering, 83 (2007)

[10] Alibas, "Characteristics of chard leaves during microwave, convective, and combined microwave - convective drying", Drying Technology, 24 (2006)

[11] C. Contreras, M. E. Martin\&Esparza, A. Chiralt and N. Martinez-Navarrete, "Influence of microwave application on convective drying: Effects on drying kinetics, and optical and mechanical properties of apple and strawberry", Journal of Food Engineering, 88 (2008)

[12] Prabhanjan, D. G., Ramaswamy, H. S. \& Raghavan, G. S. V., "Microwave-assisted convective air drying of thin layer carrots", Journal of Food Engineering, 25 (2) (1995).

[13] Andres, A., Bilbao, C., Fito, P., "Drying kinetics of apple cylinders under combined hot air-microwave dehydration", Journal of Food Engineering, 63 (2004)

[14] B. Al-Duri and S. McIntyre, Comparison of drying kinetics of food using a fan- assisted convection oven, a microwave oven and a combined microwave/convection oven, J. Food Eng., 15 (1992)

[15] J. Wang and Y.S. Xi, "Drying characteristics and drying quality of carrot using a two stage microwave process", J. Food Eng., 68 (2005)

[16] J. Wang, J. S. Wang and Y. Yu, "Microwave drying characteristics and dried quality of pumpkin", International Journal of food science and Technology, 42 (2007).

[17] Zhenfeng Li, G.S.V. Raghavan, Valérie Orsat, "Temperature and power control in microwave drying, Journal of Food Engineering", 97 (2010)

[18] Adedeji, A.A., Ngadi, M.O., Raghavan, G.S.V., "Kinetics of mass transfer in microwave precooked and deep-fat fried chicken nuggets", Journal of Food Engineering, 91 (1) (2009)

[19] Changrue, V., Orsat, V., Raghavan, G.S.V., "Osmotically dehydrated microwave-vacuum drying of strawberries", Journal of Food Processing and Preservation, 32 (5) (2008)

[20] Cui, Z.W., Xu, S.Y., Sun, D.W., Chen, W., "Temperature changes during microwave-vacuum drying of sliced carrots", Drying Technology, 23 (2005)

[21] Clary, C.D., Wang, S., Petrucci, V.E., "Fixed and incremental levels of microwave power application on drying grapes under vacuum", Journal of Food Science, 70 (5) (2005)

[22] Lu, L., Tang, J., Ran, X., "Temperature and moisture changes during microwave drying of sliced food", Drying Technology", 17 (1999)

[23] Zhang, M., Tang, J., Mujumdar, A.S., Wang, S.,"Trends in microwave-related drying of fruits and vegetables, Trends in Food \& Technology", 17 (2006),

[24] Drouzas, A. E., \& Schubert, H. "Microwave application in vacuum drying of fruits", Journal of Food Engineering, 28 (3) (1996). 
[25] Erle, U., \& Shubert, H. "Combined osmotic and microwave-vacuum dehydration of apples and strawberries", Journal of Food Engineering, 49 (2) (2001).

[26] Li, Z., Raghavan, G.S.V., Wang, N., "Carrot volatiles monitoring and control in microwave drying", LWT-Food Science and Technology (2009a)

[27] Zhenfeng Li, G.S. V. Raghavan, Ning Wang, Yvan Gariepy, "Real-time, volatile-detection-assisted control for microwave drying", Computers and Electronics in Agriculture, 69 (2009)

[28] J.I. Lombraña, R. Rodríguez, U. Ruiz," Microwave-drying of sliced mushroom. Analysis of temperature control and pressure", Innovative Food Science and Emerging Technologies, 11 (2010).

[29] D. Velić, M. Planinić, S.Tomas, M. Bilić, "Influence of airflow velocity on kinetics of convection apple drying", Journal of Food Engineering, 64 (2004)

[30] Duygu Evin, "Microwave drying and moisture diffusivity of white mulberry: experimental and mathematical modeling", Journal of Mechanical Science and Technology, 25 (10) (2011)

[31] G. Dadali and B. Ozbek, "Microwave heat treatment of leek: drying kinetic and effective moisture diffusivity", International Journal of Food Science and Technology, 43 (2008)

[32] G. P. Sharma and S. Prasad, "Effective moisture content of garlic cloves undergoing microwave - convective drying", Journal of Food Engineering, 65 (2004).

[33] H. Torgul, "Suitable drying model for infrared drying of carrot", Journal of Food Engineering, 77 (2006)

[34] Cheremisinoff, N. P., "Fundamentals of Momentum and Heat Transfer, Handbook of Heat and Mass Tranfer", Houston, Texas: Gulf, (1986)

[35] Demirel, D., \& Turhan, M., "Air-drying behaviour of Dwarf Cvendish and Gros Michel banana slices", Journal of Food Engineering, 59 (1) (2003)

[36] Iguaz, A., San Martín, M. B., Maté, J. I., Fernández, T., \& Vírseda, "Modelling effective moisture diffusivity of rough rice (Lido cultivar) at low drying temperatures", Journal of Food Engineering, 59 (2-3) (2003)

[37] Salvatori, D., Andrés, A., Chiralt, A., \& Fito, P., "Osmotic dehydration progression in apple tissue. I: Spatial distribution of solutes and moisture content", Journal of Food Engineering, 42 (1999)

[38] Cui, Z. -W., Xu, S. -Y., \& Sun, D. -W., "Microwave-vacuum drying kinetics of carrot slices", Journal of Food Engineering, 65 (2) (2004)

[39] Y.H.Hui, C.Clary, M.M.Farid, O.O.Fasina, A. Noomhorm, J. Welti-Chanes, "Food drying science and technology: Microbiology, Chemistry, Application", chapter 7.

[40] G. Cuccurullo, P. G. Berardi, "Elementi di termodinamica e trasmissione del calore", chapter 19

[41] Incropera, DeWitt, Bergman, Lavine, "Fundamentals of heat and mass transfer", chapter 14

[42] Alzamora, S., Thesis, Facultad de Ciencias Exactas y Naturales, Universedad de Buenos Aires, Argentina, 1979

[43] Labuza, T.P. and Simon, I.B., "Air drying of apples slices", Food Technology, 24 (1970)

[44] Rostein, E., Laura, P., A. and Cemborain, M.E., "Analytical prediction of drying performances in nonconventional shapes”, Journal of Food Science, 39 (1974). 